Revue

de Sémantique

et Pragmatique

\section{Revue de Sémantique et Pragmatique}

41-42 | 2017

Cadrage sur la variation, le changement lexical et le changement grammatical en français actuel

\title{
Réflexions sur les exploitations différenciées de la grammaire
}

Christophe Benzitoun, Paul Cappeau et Gilles Corminboeuf

\section{(2) OpenEdition \\ Journals}

Édition électronique

URL : http://journals.openedition.org/rsp/474

DOI : $10.4000 /$ rsp. 474

ISSN : 2610-4377

Éditeur

Presses universitaires d'Orléans

Édition imprimée

Date de publication : 1 décembre 2017

Pagination : 135-153

ISSN : $1285-4093$

Référence électronique

Christophe Benzitoun, Paul Cappeau et Gilles Corminboeuf, « Réflexions sur les exploitations différenciées de la grammaire », Revue de Sémantique et Pragmatique [En ligne], 41-42 | 2017, mis en ligne le 01 décembre 2018, consulté le 19 avril 2019. URL : http://journals.openedition.org/rsp/474 DOI : 10.4000/rsp.474 


\section{RÉFLEXIONS SUR LES EXPLOITATIONS DIFFÉRENCIÉES DE LA GRAMMAIRE}

Christophe Benzitoun, Paul Cappeau, Gilles Corminbœuf ATILF \& Université de Lorraine, FoReLL \& Université de Poitiers, FNRS \& Université de Fribourg

\section{INTRODUCTION}

e, en proposer une description unifiée, en syntaxe du moins ? Selon Gadet \& Mazière (1986 : 57), « traiter différemment oral et écrit, c'est courir le autre ne relèvent pas du même système ». La conception de l'uni

rlé' pour l'étude du 'français tout court' » (1983). Pour départager 
accourcis

iques ou revisitées

1 de réfléchir d'une

llustrent pour l'un des disparités

plus générale sur la grammaire du français.

$\mathrm{s}$ résultats issus de corpus diversifiés (en lien avec l'idée de 
s prises de parole plus

es variationnels obser

(= de manière juste ; ex. cité par Bruxelles \& al.). 
e, difficile à stocker, s'adapte en effet aussi mal

se cam

ue le rôle d'un mar

ardi gras c'était

- tout comme quand il est inverseur argumentatif. Pour Serça, 
galement

souligné que cet emploi peut apparaitre « en début de phrase ». Dans le TLFi,

.e. un « pertinentiseur », comme

ns l'oral non planifié. D'après

us oraux de parole privée ne modifient pas la 
ccurrence des écrits de linguis 
aire dans tous les corpus $(95,2 \%)$

lication pourrait être que l'emploi de marqueur de coïncidence a «basculé » dence. Cela suggère que cet emploi pragmatique est déjà ancien. 
tion extraposée finale est attestée essentiellement dans la parole

tion. Enfin, il semble parfois endosser une valeur situationnelle, réalisant une sorte d'ancrage déictique. Une typologie fine de ce que recouvre précisément la

fférentes (O-PRI) 
en relief »

» vise

utre configuration)

\begin{tabular}{|l|l|l|l|l|}
\hline & \multicolumn{2}{|l|}{} & \multicolumn{2}{|l|}{} \\
\hline & & & & \\
\hline & & & & \\
\hline & & & & \\
\hline & & & & \\
\hline
\end{tabular}


onstruction. Se pose alors la ques

é

s décrire qu'un type de production). C'est 
ns plus nettes. Jeanjean (1979) ne semble pas identifier une

être distinguées : « on admettra que 
recte (18) qui supposerait un sujet vide. 

méraux, soit aux indéfinis, piste moins visible à l'écrit. Ainsi,

ndéfinis

éfinis ont pu être observées 
és permet de mettre au jour des distributions contrastées. Si certaines

ironique lorsque l'on veut jouer sur la langue, avec la forme

de façon 
lleurs, notre conception du « genre » se révèle trop rudimentaire (Cf. Biber \& Conrad,

e référence.

Il existe enfin une variation inter-locuteurs, difficile à prendre en compte de manière méthodologiquement appropriée : par exemple, certains sujets utilisent

pos de l'oppo

ions ci-dessous. 
tiques et à dégager des structures de « langue ». (...) Je soutiens que l'objectif, désormais réalisable,

épithète en français : ce que nous ap

ilger, M. \& Cappeau, P. (2015), « Comment les données de corpus pourraient renou

our l'étude du

'français tout court' »,

2), « 
n Grammar: Where are we and where are we

going? »,

niversité de

t' pour la description des

énoncés 'fragmentés' », in

. \& Oesterreicher, W. (2001), «Langage parlé et langage écrit », in

uction il y a dans la syntaxe du français », 
torique », power-point en ligne : homepages.ulb.ac.be/ nruytenb/ website/Publications.../justement.pptx

(2012), « La séduction du binaire ». 